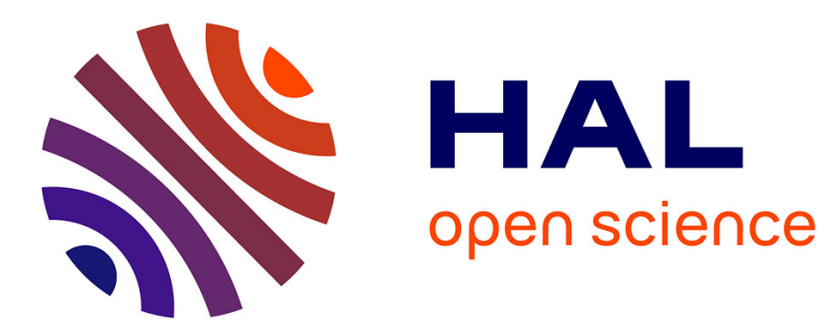

\title{
Sound of effervescence
}

Mathis Poujol, Régis Wunenburger, François Ollivier, Arnaud Antkowiak, Juliette Pierre

\section{To cite this version:}

Mathis Poujol, Régis Wunenburger, François Ollivier, Arnaud Antkowiak, Juliette Pierre. Sound of effervescence. Physical Review Fluids, 2021, 6 (1), 10.1103/PhysRevFluids.6.013604 . hal-03121607

\section{HAL Id: hal-03121607 https://hal.science/hal-03121607}

Submitted on 22 Feb 2021

HAL is a multi-disciplinary open access archive for the deposit and dissemination of scientific research documents, whether they are published or not. The documents may come from teaching and research institutions in France or abroad, or from public or private research centers.
L'archive ouverte pluridisciplinaire HAL, est destinée au dépôt et à la diffusion de documents scientifiques de niveau recherche, publiés ou non, émanant des établissements d'enseignement et de recherche français ou étrangers, des laboratoires publics ou privés. 


\title{
Sound of effervescence
}

\author{
Mathis Poujol, Régis Wunenburger, François \\ Ollivier, Arnaud Antkowiak, and Juliette Pierre* \\ Sorbonne Université, CNRS, Institut Jean le Rond d'Alembert, F-75005 Paris, France
}

(Dated: March 4, 2021)

\begin{abstract}
Capillary bubbles burst at a free surface following a rapid sequence of events occurring at different length- and timescales: hole nucleation, fast retraction of the micron-thick liquid film in a few microseconds preluding the much slower overall collapse of the millimeter-sized bubble in a matter of milliseconds. Each of these steps is associated with unsteady fluid forces and accelerations, and therefore with sound radiation. In this experimental study we focus on the airborne sound generated during bubble bursting. Investigating the physical mechanism at the root of sound emission with the help of synchronized fast imaging and sound recordings, we quantitatively link the film retraction dynamics with the frequency content of the acoustic signal. We demonstrate that, contrary to a Minnaert resonance scenario, the frequency here drifts and increases, consistently with a Helmholtz-type resonance of the cavity being more and more opened as the thin film retracts. We propose as an extension a simple model based on a collection of drifting Helmholtz resonators capturing the main features of the fizzing sound of an effervescing beverage.
\end{abstract}

\footnotetext{
* juliette.pierre@sorbonne-universite.fr
} 


\section{INTRODUCTION}

The crackling and fizzing sound of a freshly served glass of champagne is a matter of common experience (see Fig. 1(a)). While there is little doubt that the sound originates from the bursting of capillary bubbles, the physical mechanism and overall picture of sound emission remains still somewhat unclear. In this work we propose to combine high-speed imaging with synchronous acoustic signal recordings in order to decipher the link between the fluid dynamics of bubble bursting and acoustic emission, and to pinpoint the physical mechanism responsible for the sound of effervescing beverages.

A typical acoustic signal emitted in the air during $1 \mathrm{~s}$ by an effervescent glass of champagne is shown in Figure 1(c) and a $5 \mathrm{~ms}$ duration part of it are shown in Figure 1(d). It displays a succession of sine-like short emissions (tone bursts). Processing the images of the liquid surface synchronised with the acoustic signal shown in Figure 1(e-h) reveals that a tone burst is emitted concomitantly with a collapsing bubble event occurring at the liquid surface. Further, the Fourier spectrum of the overall fizzing sound presents a striking similitude with that of a single bursting event (Fig. 1(i)), thereby strongly suggesting that the crackling sound of a glass of champagne merely results from a superposition of tone bursts emitted by single bubble bursts.

Champagne bubbles have been intensively studied [1-4]. Champagne and more generally sparkling beverages are saturated with $\mathrm{CO}_{2}$ and their effervescence usually originates from the nucleation of bubbles on the glass wall. After detaching from their nucleation sites whose size can be as small as $1 \mu \mathrm{m}$, bubbles grow in size due to gas diffusion during their rise to the liquid surface. At the surface where they ultimately collapse, their equivalent radius $R$, defined as

$$
R=\left(\frac{3 V}{4 \pi}\right)^{1 / 3}
$$

where $V$ is the bubble volume, ranges between $100 \mu \mathrm{m}$ and $500 \mu \mathrm{m}$, as shown in Fig. 1(b) [5]. In contrast with the extensive knowledge on the physics and chemistry of effervescence [1,2], only one study dedicated to the acoustic emission of champagne could be identified, in which the sound was related to the bubble size distribution [6].

The Bond number is the dimensionless quantity measuring the relative contribution of

gravity and capillarity to the bubble shape: $\mathrm{Bo}=\left(R / \ell_{c}\right)^{2}$, where $\ell_{c}=\left(\sigma / \rho_{l} g\right)^{1 / 2}$ is the capillary length $\left(\rho_{l}\right.$ is the liquid density, $\sigma$ the liquid surface tension and $g$ the gravity 
acceleration). For champagne bubbles, $\ell_{c}=2.2 \mathrm{~mm}$ (see Table I), which results in Bo « 1 (for our experiments Bo falls in the range $[2 ; 50] \times 10^{-3}$ ) and thus in nearly spherical bubbles. Besides, we also note that champagne usually contains surfactant molecules [4]
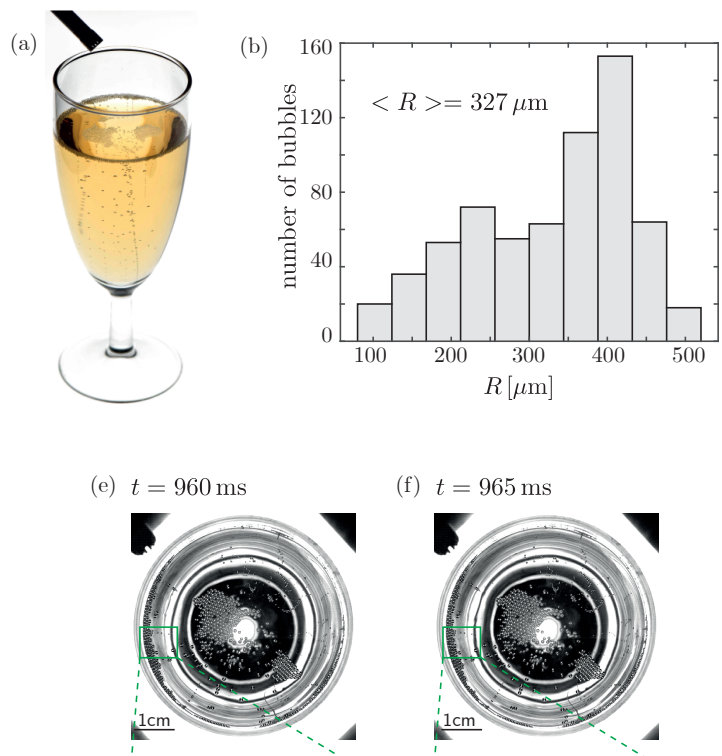

(g)

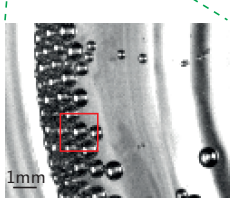

(f) $t=965 \mathrm{~ms}$

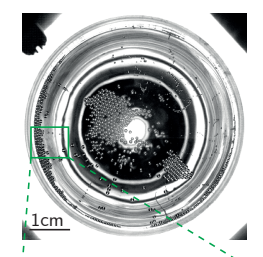

(h)

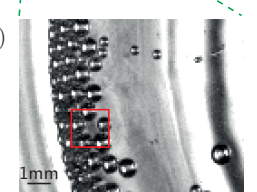

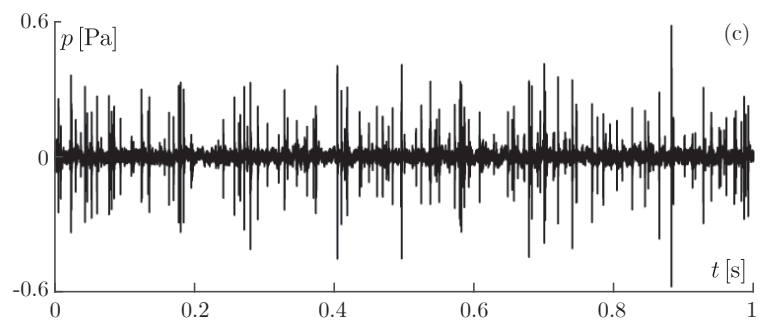
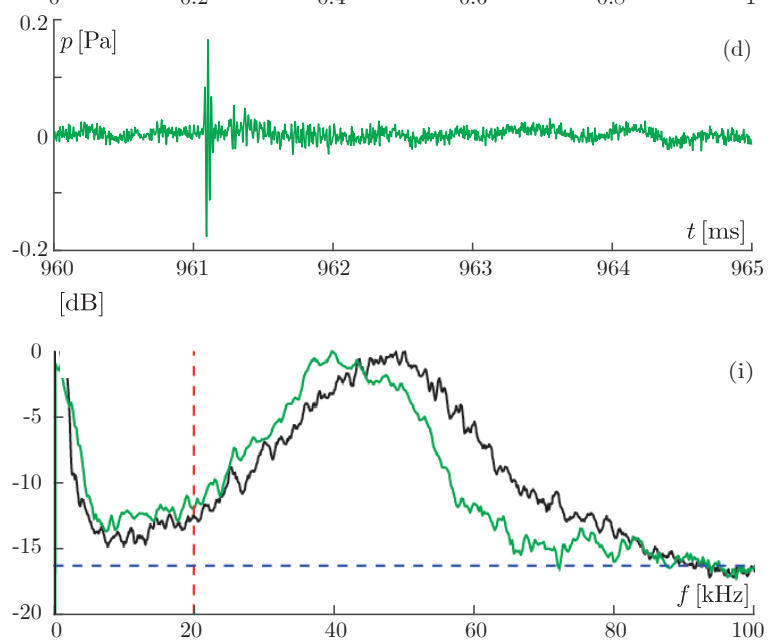

FIG. 1. Simultaneous acquisition of the airborne sound emitted by champagne in a glass and of a movie of the liquid surface viewed from the top. (a) Picture of a glass of champagne showing the microphone placed a few centimetres above the glass edge. (b) Histogram of the bubble radii extracted from a picture of the liquid surface at time $t=960 \mathrm{~ms}$ average radius $<R>=327 \mu \mathrm{m}$ ).(c) 1 s-duration time history of the acoustic pressure high-pass filtered with a $50 \mathrm{~Hz}$ cut-off frequency and a sampling frequency of $500 \mathrm{kHz}$. (d) 5 ms-duration part of the signal centred on a single emission event occurring at $t=961 \mathrm{~ms}$. (e-h) Top view pictures of the liquid surface acquired at 200 fps using a high-speed camera synchronised with the acoustic signal acquisition. Pictures $(\mathrm{e}, \mathrm{f})$ and the enlargement of their framed part $(\mathrm{g}, \mathrm{h})$ evidence the disappearance of one bubble inside the red frame between $t=960 \mathrm{~ms}$ and $t=965 \mathrm{~ms}$. (i) Acoustic spectra of the $1 \mathrm{~s}$-duration acoustic recording (c) (black curve) and of its $5 \mathrm{~ms}$ duration enlargement (d) (green curve). The blue horizontal dashed line indicates the noise level and the vertical dashed line indicates the limit between the audible domain and the ultrasound domain. 
at low concentrations. In this paper we therefore study the sound radiated in the air by single capillary bubbles collapsing at the surface of both a pool of tap water and of watersurfactant solution (whose properties are given in Table I), the latter being used as a proxy for champagne to build an understanding of champagne sound emission.

Floating capillary bubbles are almost entirely submerged and are separated from the atmosphere by a thin liquid film. Mechanical equilibrium implies that the bubbles are overpressurised by the Laplace pressure $\Delta P \sim 2 \sigma / R$. Due to gravity drainage and capillarity suction, the film becomes thinner and thinner and finally bursts, as sketched in Fig. 2 (a, b) and shown in Fig. 2 (c). The film bursting leads to the sudden release of the internal pressure, which triggers the bubble collapse, see Fig. 2 (d). High-speed side views of capillary air bubbles collapsing at the surface of a water pool reveal that this event lasts a few milliseconds and involves several steps. First, the liquid film breaks and retracts over a typical $100 \mu$ s duration, as shown in Fig. 2 (c). The cavity collapse occurs over a larger timescale, typically a couple of milliseconds, as shown in Fig. 2 (d). Eventually the cavity reverses and a liquid jet is ejected, possibly rupturing into droplets and/or aerosols. These hydrodynamic processes are still the subject of a recent and active research, see e.g. [8-11].

Here we acquire a high-speed chronophotography of this bubble collapse simultaneously with its emitted acoustic signal. As shown in Fig. 2 (e), the bubble emits sound during the first $200 \mu \mathrm{s}$ after the onset of film retraction. The acoustic emission of capillary bubbles collapsing at a free water surface was studied in Spiel [12] and Deane [13]. Their

\begin{tabular}{|l|l|l|l|l||l|l|l|}
\hline Liquid solution & density & viscosity & equilibrium \\
$\rho_{l}\left(\mathrm{~kg} \mathrm{~m}^{-3}\right)$ & $\mu(\mathrm{mPa} \mathrm{s})$ & $\begin{array}{l}\text { capillary } \\
\text { surface } \\
\text { length } \\
\text { tension } \\
\sigma\left(\mathrm{mN} \mathrm{m}^{-1}\right)\end{array}$ & $\begin{array}{l}\text { Gas } \\
\ell_{c}(\mathrm{~mm})\end{array}$ & $\begin{array}{l}\text { density } \\
\rho_{g}\left(\mathrm{~kg} \mathrm{~m}^{-3}\right)\end{array}$ & $\begin{array}{l}\text { celerity } \\
c\left(\mathrm{~m} \mathrm{~s}^{-1}\right)\end{array}$ \\
\hline Tap water & $10^{3}$ & 1 & 72 & 2.7 & air & 1.2 & 345 \\
\hline $\begin{array}{l}\text { SDS at } 2 \mathrm{~g} \mathrm{~L}^{-1}+ \\
\text { distilled water }\end{array}$ & $10^{3}$ & 1 & 35 & 1.9 & air & 1.2 & 345 \\
\hline $\begin{array}{l}\text { Champagne } \\
\text { (from [4]) }\end{array}$ & 992 & 1.6 & 48 & 2.2 & $\begin{array}{l}\mathrm{CO}_{2} \\
(\text { from }[7])\end{array}$ & 1.8 & 267 \\
\hline
\end{tabular}

TABLE I. Properties of the liquids and gases used in this work at $22^{\circ} \mathrm{C}$. 
acoustic emission was interpreted as the transient acoustic emission associated to the free, damped oscillations of a Helmholtz resonator following the instantaneous release of its initial over-pressure. The central frequency of the oscillations of the acoustic signal was found to quantitatively agree with the Helmholtz resonator model [12]. Besides, it was observed that the signal emitted by the smallest bubbles $(R<1 \mathrm{~mm})$ was chirped [12]. The origin of this phenomenon is still unclear to date, even if film retraction was speculated to play a role in Spiel [12] and Deane [13] - yet without providing any experimental analysis or data on film retraction. More precisely, Spiel [12] conducted the analysis of the frequency modulation

(a)

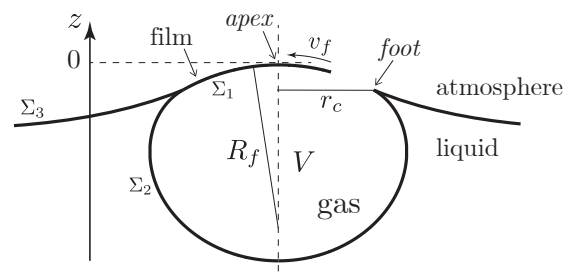

(b)
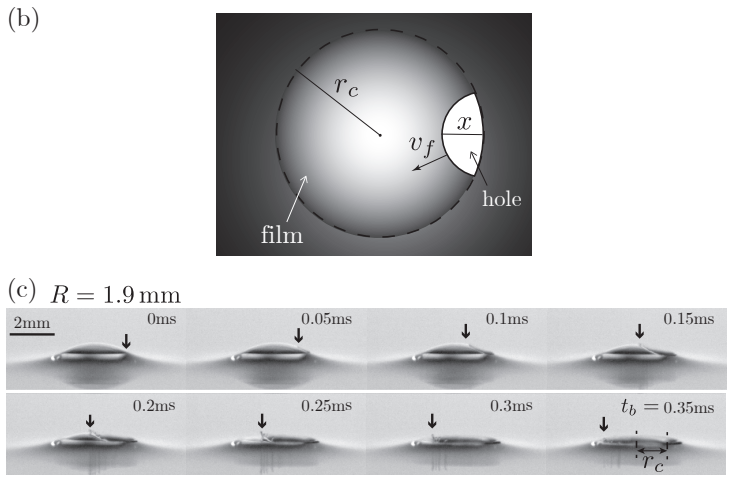
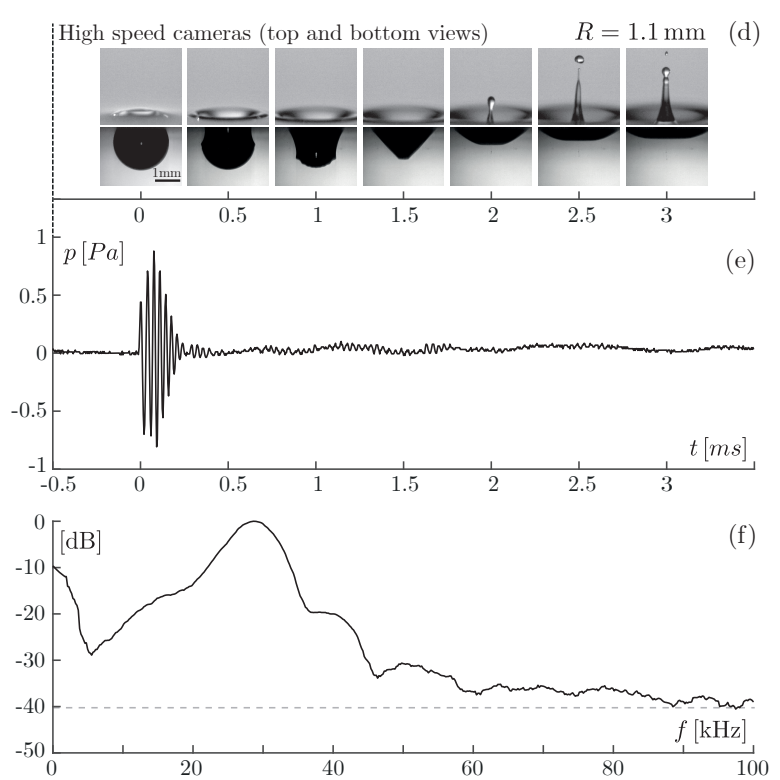

FIG. 2. Sketch of a floating bubble at the initial stage of its collapse corresponding to the retraction of the liquid film: (a) profile view, (b) top view. (c) Sequence of pictures of a floating air bubble with $R=1.9 \mathrm{~mm}$ equivalent radius viewed from the side above the free water surface, showing the retraction of the water film separating the bubble from the atmosphere. The black arrow indicates the position on the rim of the retracting film. (d) Sequence of pairs of pictures of a $R=1.1 \mathrm{~mm}$ collapsing air bubble viewed from the side above and below the free water surface using two synchronised high-speed cameras. (e) Synchronised airborne acoustic pressure signal emitted during the collapse and detected using a microphone located $20 \mathrm{~mm}$ right above the bubble. (f) Fourier spectrum of pressure signal (dashed line: noise level) (Normalised by the maximum of the spectrum). 
after the end of the film retraction, while Deane [13] indirectly determined the film thickness, key in retraction, from its draining dynamics. Time-resolved imaging of the bubble collapse therefore appears as a mandatory complement to acoustic monitoring to definitively clarify the origin of bubble bursting sound emission.

\section{EXPERIMENT}

Air bubbles are generated in a parallelipedal glass tank $(20 \mathrm{~cm}$ length, $14 \mathrm{~cm}$ width, $9.5 \mathrm{~cm}$ depth) filled with either tap water or an aqueous solution of SDS (Sodium Dodecyl sulfate

- purchased from Sigma Aldrich) surfactant with $2 \mathrm{~g} / \mathrm{L}$ mass concentration, i.e. $0.9 \times c_{\mathrm{cmc}}$, where $c_{\mathrm{cmc}}$ is the critical micellar concentration (see Tab. I). Bubbles are released from submerged needles connected to a syringe pump filled with air. The needle internal diameters range from $0.08 \mathrm{~mm}$ to $1.5 \mathrm{~mm}$. The bubbles rise to the water surface and briefly float before bursting.

Two digital high speed cameras (Photron SA-5 and Phantom V2511 types) are used to image the collapsing bubble from the side, one below the free surface, one above it, see Fig. 2 (d). The airborne acoustic signature of the bubble collapse is recorded using a Brüel \& Kjær 4939-A-011-type microphone with [4 Hz - $100 \mathrm{kHz}]$ frequency range at least at $500 \mathrm{kHz}$ sampling frequency. The microphone is positioned at a distance $d=20 \mathrm{~mm}$ above the free liquid surface, right above the floating bubble and is oriented vertically downward. The audio and video recordings are synchronised. The acoustic signals are then time-shifted by the time-of-flight of sound between the surface and the microphone, $58 \mu$ s. Finally, the acoustic signals are high-pass filtered at $50 \mathrm{~Hz}$ cut-off frequency in order to remove most of the ambient noise principally due to air-stream from cameras, electronic devices and room ventilation. Note that we measured no influence of the tank size on the acoustic signals. Noticeably, each bubble collapse event is thoroughly analyzed so that all the quantities reported in the following figures are extracted from each event.

\section{DESCRIPTION OF THE FLOATING BUBBLE}

To evaluate the volume $V$ of the floating bubble, we follow the procedure implemented in the work of Ghabache et al [9]. First, we image the floating bubble from the side below 
the surface using a high-speed camera just before the collapse onset. A typical picture of a bubble is shown in Fig. 3(a). The bubble lower hemisphere being regarded as an ellipsoid with vertical symmetry axis, we determine the best fit of its profile by an ellipse with semiminor axis $a$ and semi-major axis $b$. The bubble volume is assumed to be equal to the volume of the corresponding ellipsoid $V=\frac{4}{3} \pi a b^{2}$ and the bubble equivalent radius is $R=\left(a b^{2}\right)^{1 / 3}$. The radii of the studied bubbles range from $0.3 \mathrm{~mm}$ to $3 \mathrm{~mm}$.

Since Bo $\lesssim 1$, floating bubbles are not spherical. Assuming the floating bubbles to be at hydrostatic equilibrium, their shape and altitude are determined by the balance between surface tension and buoyancy. Floating bubbles display the following features sketched in Fig. 2 (a): (i) an ellipsoid-like liquid-gas interface below the free surface, called $\Sigma_{2}$, (ii) a spherical shaped liquid film between the gas bubble and the atmosphere at the bubble top, called $\Sigma_{1}$ and having the radius of curvature $R_{f}$.

The liquid film being considered as infinitely thin, $R_{f}$ satisfies the Young-Laplace equation

$$
\Delta P=\frac{4 \sigma}{R_{f}},
$$

where $\Delta P$ is the air over-pressure inside the bubble, considered as homogeneous. $z$ being the altitude and the film highest point being at altitude $z=0$ (see Fig. 2 (a)), the curvature $\kappa_{2}(z)$ of the submerged liquid-air interface $\Sigma_{2}$ satisfies

$$
\Delta P=\sigma \kappa_{2}(z)-\rho_{l} g z
$$

and the curvature $\kappa_{3}(z)$ of the free surface surrounding the bubble satisfies

$$
\sigma \kappa_{3}(z)-\rho_{l} g z=0
$$

The set of equations (2), (3) and (4) constrained by the conditions of fixed air volume $V$ and flat free surface far away from the bubble fully determines $\Delta P$; it is called hereafter the Young-Laplace problem.

In the Bo $\ll 1$ limit, capillary effects are predominant over gravity effects. Thus, the pressure in water can be considered as homogeneous across the bubble and both the upper liquid film and the liquid-gas interface below the free surface can be considered as spherical. Thus, $\Delta P$ satisfies two simplified Young-Laplace equations $\Delta P=4 \sigma / R_{f}$ and $\Delta P=2 \sigma / R$, which result in $R_{f}=2 R$.

The numerical resolution of the Young-Laplace problem following the method described in the work of Toba [14] using $V$ as input allows us to determine $\Delta P$ and the bubble shape, in 
particular $R_{f}$ and the radius $r_{c}$ of the circular edge of the liquid film $\left(\Sigma_{2}\right)$ viewed from above, see Fig. 3(a). The solution of the Young-Laplace problem, shown in Fig. 3(a), is found to coincide with the bubble profile along $\Sigma_{1}, \Sigma_{2}$ and $\Sigma_{3}$. This demonstrates the accuracy of the determination of $V$ using an elliptic fit of the bubble profile. The corresponding variation of $r_{c}$ versus $R$ for capillary bubbles is shown in the inset of Fig. 3(b). The law proposed in Lhuissier and Villermaux [15] in the $\mathrm{Bo} \rightarrow 0$ limit

$$
r_{c}=\frac{2}{\sqrt{3}} \frac{R^{2}}{\ell_{c}}
$$

quantitatively fits this curve in the Bo $\ll 1$ range, as shown in the inset of Fig. 3(b).

To check the validity of this law, we measure $r_{c}$ by exploiting the pictures of the film opening. Since the rim of the retracting film is clearly visible in these pictures, as shown in Fig. 2 (c), its disappearance indicates that it has reached the film edge, whose distance to the bubble axis is equal to $r_{c}$. The variations over more than a decade of the experimental values of $r_{c}$ are compared to their theoretical prediction in Fig. 3(b). Given experimental scatter, a good agreement is observed, which confirms the validity of the model of bubble shape presented in this section.

\section{DYNAMICS OF THE FILM OPENING}

In the case of pure water, i.e. surfactant-free films, the lifetime of floating bubbles is very short, usually shorter than $1 \mathrm{~s}$, and the films always break at the bubble foot and not at the apex, as reported in Lhuissier [15] and Champougny [16]. In the case of SDS solutions, the lifetime of floating bubbles is much longer, usually of the order of $1 \mathrm{~min}$, and the film breaking takes place at or close to the bubble top.

We define the film opening duration $t_{b}$ as the time interval between the picture on which the rim appears at a point located at the bubble foot and the picture on which the rim reaches the diametrically opposed point and disappears, see Fig. 2 (c). The variation of $t_{b}$ versus $R$ for water and SDS solutions is shown in log-log scales in Fig. 4 (a). $t_{b}$ ranges between $10 \mu \mathrm{s}$ and $500 \mu \mathrm{s}$ in the investigated range of values of $R$.

In the case of pure water, since the film breaks at the bubble foot, (i) the hole opening dynamics results from the retraction of a single rim, as sketched in Fig. 2 (a, b) and (ii) the film rim travels a total distance equal to $2 R_{f} \arcsin r_{c} / R_{f} \simeq 2 r_{c}$, neglecting the film 

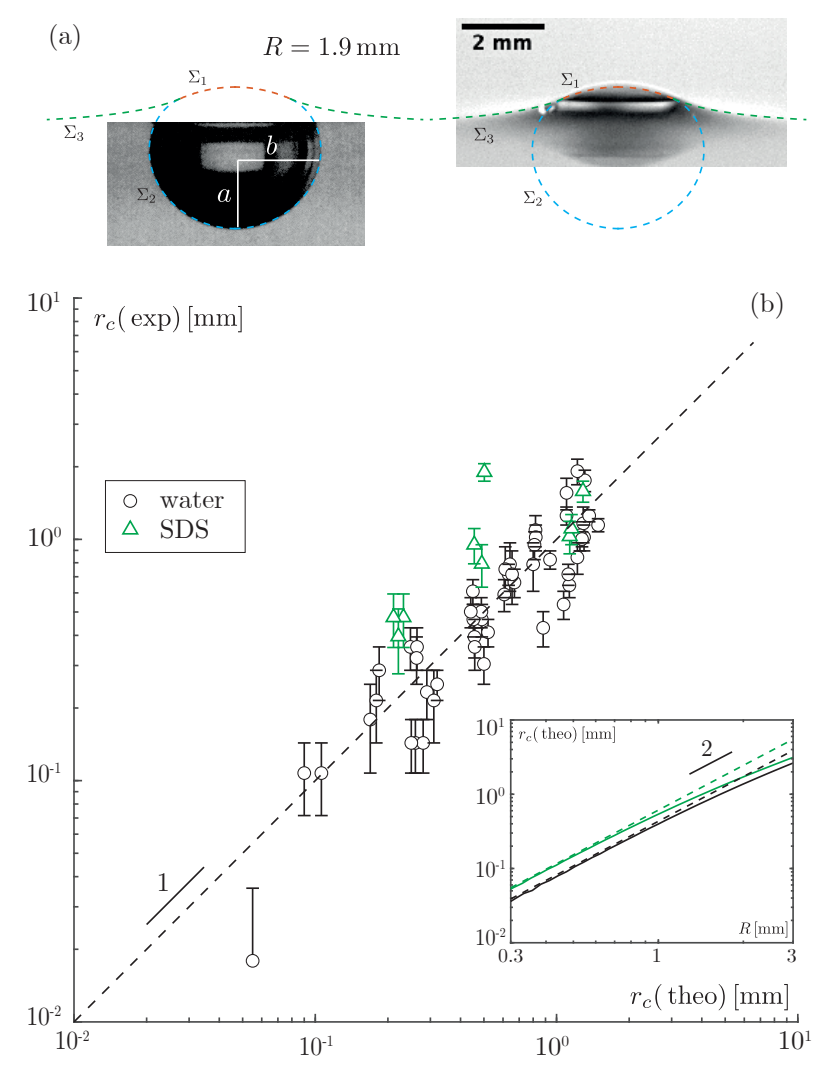

FIG. 3. (a) Pair of pictures from the side below (left) and above (right) the surface of a $R=1.9 \mathrm{~mm}$ air bubble floating at a water free surface. $\Sigma_{1}$ : liquid film separating the inner air from the atmosphere. $\Sigma_{2}$ : water-air interface below the free surface. $\Sigma_{3}$ : free liquid surface. a (respectively b): semiminor (respectively semimajor) axis of the elliptic best fit of the bottom of the bubble profile. Red (respectively blue, green) dashed curve: shape of $\Sigma_{1}$ (respectively $\Sigma_{2}, \Sigma_{3}$ ) solution of the Young-Laplace problem (see text). (b) Measured $r_{c}$ versus their theoretical prediction. The solid line corresponds to the perfect agreement between experiment and theory. Inset: theoretically predicted variation of $r_{c}$ versus $R$ for pure water (black solid curve) and SDS solution (green solid curve) together with Eq. (5) for pure water (black dashed curve) and SDS solution (green dashed curve).

curvature, as shown in Fig. $2(\mathrm{a}, \mathrm{b})$. The distance $x$ travelled by the rim at time $t$ since film breaking, sketched in Fig. 2 (a) is represented in Fig. 4(b) for several values of $R$. In a first stage, $x$ varies linearly with time, which indicates that the rim retraction velocity is constant and of the order of $10 \mathrm{~m} \mathrm{~s}^{-1}$. Liquid films with thickness $h$ are known to retract at 
velocity $v_{f}$ such that

$$
v_{f}=\sqrt{\frac{2 \sigma}{\rho_{l} h}}
$$

as the result of the balance between the inertia of the growing rim and the capillary forces exerted by the liquid film on the rim $[17,18]$. Since this linear behaviour of $x(t)$ is always observed, i.e. $x(t)=v_{f} t$, we conclude that the thickness $h$ of the spontaneously opening films is always homogeneous. Consequently, $h$ can be deduced from $v_{f}$ by inverting Eq. (6). The variation of $h$ versus $R$ is shown in the inset of Fig. 4(b). $h(R)$ displays no trend but rather a scatter characterised by a $1.6 \mu \mathrm{m}$ standard deviation around an average value $\langle h\rangle=2.7 \mu \mathrm{m}$ for water bubbles.

Given that $v_{f}$ is constant during each film opening, $t_{b}$ satisfies

$$
t_{b} \simeq \frac{2 r_{c}}{v_{f}}
$$

Since $v_{f}$ does not depend on $R$, in the following we use its average value $\left\langle v_{f}\right\rangle$. By combining Eqs. (5), (6) and (7), we can express $t_{b}$ as :

$$
t_{b}=\frac{4}{\sqrt{6}} \frac{\sqrt{g\langle h\rangle} \rho_{l} R^{2}}{\sigma} .
$$

This law is represented in Fig. 4(a) for water and SDS solutions (Eq. (6) has been shown to remain valid for retracting films made of SDS solutions as long as $h \geq 0.1 \mu \mathrm{m}$ [19]). Given the experimental scatter, a good agreement is observed, which confirms the validity of the model of the film opening dynamics presented in this section.

As shown in Fig. 4(b), as soon as $x>2 r_{c}, x$ still varies linearly with time but with a much smaller velocity (of the order of $1 \mathrm{~m} \mathrm{~s}^{-1}$ ). This corresponds to the receding motion of the upper edge of the cavity, i.e. to the junction between surfaces $\Sigma_{2}$ and $\Sigma_{3}$.

In the case of SDS solution, since the film breaks at or close to the bubble top, the hole is circular during most of the opening duration and consequently the hole diameter, also called $x$ here for simplificity, satisfies $x(t)=2 v_{f} t$. Thus, $t_{b}=r_{c} / v_{f}$.

These results on the hydrodynamic evolution of a collapsing bubble constitute the background for analysing its acoustic emission. 


\section{ACOUSTIC EMISSION}

\section{A. Characteristic features of the acoustic emission}

A typical airborne acoustic pressure signal emitted during the collapse of an air bubble floating at the water free surface is shown in Fig. 5 (a). The spectrogram of the signal shown in Fig. 5 (a) is plotted in Fig. 5 (b). We observe that the instantaneous spectra are peak-shaped at all times and that the spectrogram displays two regimes: a first regime during which the corresponding peak frequency $f_{0}$ increases with time, i.e. during which the signal is chirped, and a second regime during which $f_{0}$ appears as approximately constant.

The variations of $f_{0}$ with time for several values of $R$ are represented in log-log scales in Fig. 6 (a). In the first regime, $f_{0}$ is observed to approximately agree with a $t^{1 / 2}$ scaling law, while in the second regime, $f_{0}$ increases much more slowly. We define $\tau_{b}$ as the time at which the transition between the two regimes occurs, see Fig. 6 (a). $\tau_{b}$ is called the chirp
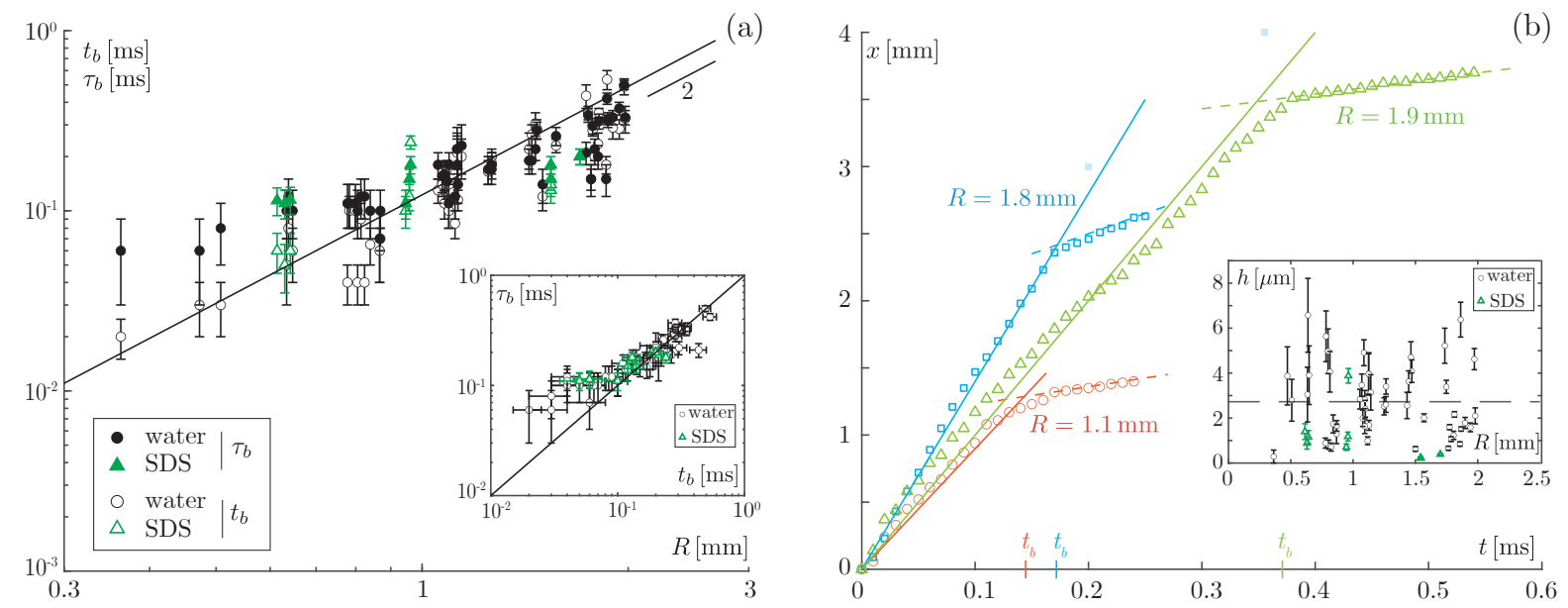

FIG. 4. (a) Measured film opening duration $t_{b}$ and measured duration $\tau_{b}$ of the frequency chirp of the acoustic emission versus $R$. Black line: theoretical prediction Eq. (8) for $t_{b}$ corresponding to the average value $\langle h\rangle=2.7 \mu \mathrm{m}$ of the retracting liquid films. Inset: measured duration $\tau_{b}$ of the frequency chirp of the acoustic emission versus measured film opening duration $t_{b}$. (b) Timedependent distance $x$ travelled since film breaking by the rim (up to $t_{b}$ ), then by the $\Sigma_{2}-\Sigma_{3}$ junction $\left(\right.$ from $t_{b}$ ) for three different values of $R$. Lines are best linear fits. Inset: thickness $h$ of the retracting liquid films for the water bubbles deduced from Eq. (6) versus $R$. Dashed line: average value of $h$ for water bubbles. 
duration hereafter. The variation of $\tau_{b}$ with $R$ is represented in Fig. 4 (a). In the inset we also compare $\tau_{b}$ with the film opening duration $t_{b} . t_{b}$ is observed to coincide with $\tau_{b}$ for bubbles larger than $0.5 \mathrm{~mm}$ given the experimental scatter. This demonstrates that the chirp is correlated with the film opening. Moreover, the influence of the bubble geometry on its acoustic emission has to be questioned.

\section{B. Helmholtz's resonator model}

In the works of Spiel [12] and Deane [13], the acoustic emission of collapsing bubbles was modelled using Helmholtz's model of airborne radiation of an open cavity [20]. According to this lumped-element model of open acoustic resonator, a cavity with volume $V$ communicating with the atmosphere through an bottleneck-like aperture with area $S$ and length $L$ behaves as a mass-spring harmonic oscillator whose inertia is associated to the

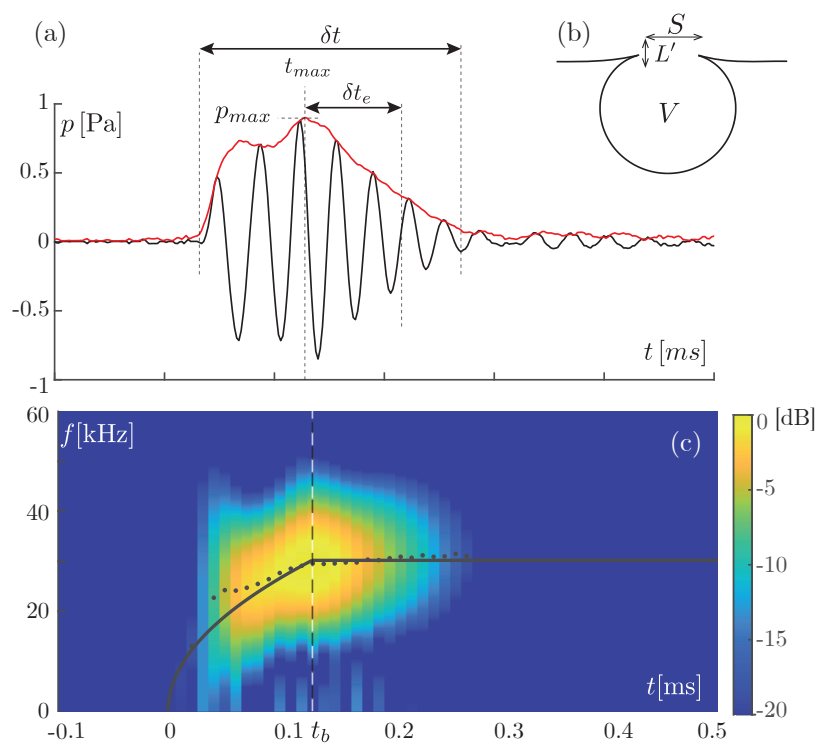

FIG. 5. (a) Airborne acoustic pressure signal emitted during the collapse of a $R=1.1 \mathrm{~mm}$ air bubble floating at a water free surface. Red curve: absolute value of the Hilbert transform of the signal. (b) Sketch of the opening bubble. (c) Spectrogram of the signal shown in (a). Dots: maxima of the instantaneous spectra. The vertical dashed line indicates the time $t_{b}$ defined by Eq. (8) at which the film retraction is completed. Black solid curve: prediction of $f_{H}$ based on Eqs. (11), (13), (12), (8). 

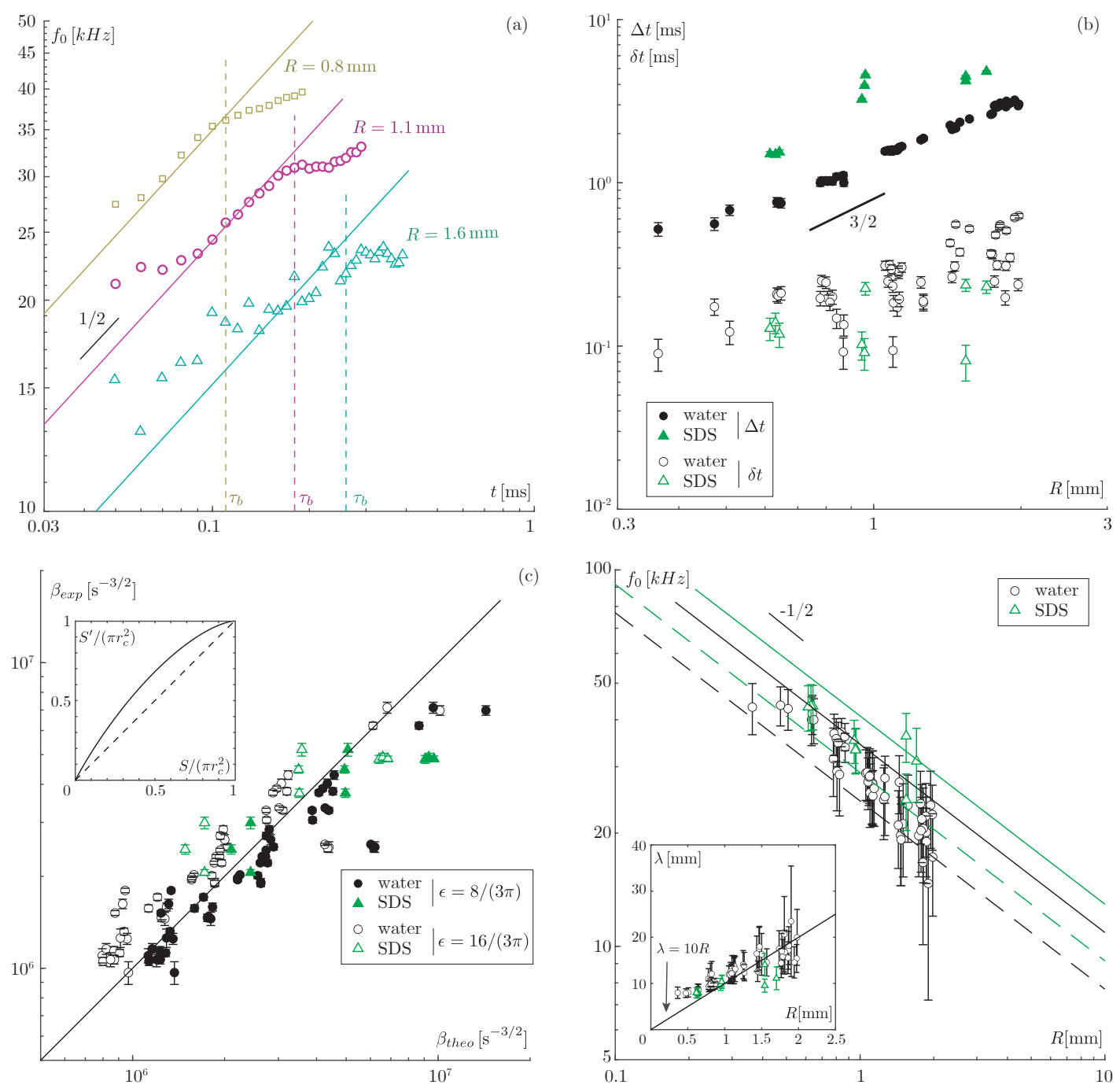

FIG. 6. (a) Time-dependent peak frequency $f_{0}(t)$ of the instantaneous spectra of the airborne acoustic emission of collapsing bubbles for three different values of the bubble equivalent radius $R$. (b) Measured duration $\delta t$ of the acoustic signal and duration of the bubble collapse $\Delta t$. Solid line: scaling law for $\Delta t$ from [9]. (c) Prefactor of the scaling law $f_{H}=\beta_{\exp } t^{1 / 2}$ as a function of its theoretical prediction $\beta_{\text {theo }}$ using by Eq. (12). The solid line corresponds to the perfect agreement between experiment and theory. Inset: Variation of the section $S^{\prime}$, defined in eq. 10, over the variation of area $S$ defined for a circular hole. (d) Variation versus $R$ of the peak frequency $f_{0}\left(t_{b}\right)$ of the instantaneous spectra of the airborne acoustic emission of collapsing bubbles at the instant $t_{b}$ at which the liquid film opening is ending. Black line (respectively dashed line): theoretical prediction Eq. (13) with $\varepsilon=8 /(3 \pi)$ (respectively $\varepsilon=16 /(3 \pi))$. Inset: acoustic wavelength $\lambda$ versus $R$. Solid line: law $\lambda=10 R$. 
mass of the air column oscillating in the cavity aperture and whose stiffness is associated to the compressibility of the air enclosed in the cavity. The corresponding oscillator resonant frequency $f_{H}$ is such that

$$
f_{H}=\frac{c}{2 \pi} \sqrt{\frac{S}{L^{\prime} V}}
$$

where $c$ is the speed of sound in the gas, $L^{\prime}=L+\varepsilon r$ the length of the air column oscillating in the cavity aperture, $r$ the radius of the aperture regarded as circular and $\varepsilon$ a dimensionless parameter reflecting the nonlinear air flow inside and outside the aperture, typically $\varepsilon=8 /(3 \pi)$ in the case of a non-baffled resonator and $16 /(3 \pi)$ in the case of a baffled resonator [20]. This model can in principle be applied to a collapsing bubble by considering that the cavity corresponds to the bubble volume and the aperture to the hole in the liquid film, both varying during the collapse.

Since the cavity starts collapsing during its acoustic emission, we might wonder if the bubble volume can be assumed as constant during the bubble acoustic emission. To answer this question, we compare the duration of the acoustic emission $\delta t$ to the collapse duration $\Delta t . \Delta t$ is defined as the time elapsed between the hole nucleation and the cavity reversal (i.e. when the depth of the immersed cavity starts to increase - close to $1.5 \mathrm{~ms}$ in Fig.2 (d)). The variation of $\Delta t$ versus $R$ is plotted in Fig. $6(\mathrm{~b})$. We observe that $\Delta t$ scales approximately as $R^{3 / 2}$, in agreement with the scaling law $\Delta t \sim \sqrt{\rho R^{3} / \sigma}$ describing the inertial collapse of the cavity driven by surface tension [9]. $\Delta t$ increases when SDS-surfactant is used because $\Delta t$ scales as $\sigma^{-1 / 2}$ and $\sigma$ is lower in presence of surfactant. On the other hand, $\delta t$ is defined as the time elapsed during which the amplitude of the Hilbert transform of the pressure signal is larger than $p_{\max } / 10$, where $p_{\max }$ is the maximum of the Hilbert transform, see Fig. 5 (a). The variation of $\delta t$ versus $R$ is plotted in Fig. 6 (b). We observe that $\delta t<\Delta t$ in the whole range of the investigated values of $R$ (interestingly, $\delta t$ exhibits a large dispersion but the upper boundary of the data exhibits the same scaling as $\Delta t$ ). We conclude that the acoustic emission proceeds and ends at the very beginning of the bubble collapse. Consequently, we can safely assume the bubble volume to be constant and equal to its initial volume $V$ during its acoustic emission.

Due to the geometry of the opening bubble sketched in Fig. 5 (a), the length $L$ of the resonator aperture can be taken equal to zero. In the case of surfactant-free water, since the liquid film breaks at the bubble foot, the hole is not circular, as sketched in Fig. 5 (b). 
Therefore, we cannot straightforwardly define an equivalent aperture radius $r$. But the area $S^{\prime}$ of a hole with width $x$,

$$
S^{\prime}=2\left(\frac{x^{2}}{2} \arccos \left(\frac{x}{2 r_{c}}\right)+r_{c}^{2} \arcsin \left(\frac{x}{2 r_{c}}\right)\right)-x \sqrt{r_{c}^{2}-\left(\frac{x}{2}\right)^{2}}
$$

is not very different from the area $S=\pi(x / 2)^{2}$ of a circular hole with radius $x / 2$, as shown in the inset of Fig. 6 (c). Consequently, in the following we approximate $S^{\prime}$ by $S$ and we assume the equivalent aperture radius $r$ associated to the film hole to equal $x / 2$ and $L^{\prime} \simeq \varepsilon x / 2$.

With these approximations, the resonant frequency $f_{H}$ of the acoustic emission of the collapsing bubble satisfies:

$$
f_{H}(t)=\beta_{\text {theo }} t^{1 / 2} \text { for } t \leq t_{b}
$$

with

$$
\beta_{\text {theo }}=\frac{c}{4 \pi} \sqrt{\frac{3 v_{f}}{2 \varepsilon R^{3}}}
$$

Noticeably, at $t=t_{b}$,

$$
f_{H}\left(t_{b}\right)=\beta_{\text {theo }} t_{b}^{1 / 2}=\frac{c}{4 \pi} \sqrt{\frac{2 \sqrt{3}}{\varepsilon \ell_{c} R}} .
$$

According to Eq. (11), $f_{H} \propto t^{1 / 2}$, which is in agreement with the experimental observation that $f_{0} \propto t^{1 / 2}$. To quantitatively test the validity of Eq. (11), we determine the best fit of $f_{0}(t)$ by the function $\beta_{\exp } t^{1 / 2}$ with $\beta_{\exp }$ as free parameter, see Fig. 6 (a). The variation of $\beta_{\exp }$ as function $\beta_{\text {theo }}$ computed using $\varepsilon=8 /(3 \pi)$ and $\varepsilon=16 /(3 \pi)$ are plotted in Fig. 6 (c). Given the experimental scatter, a satisfactory correlation between $\beta_{\exp }$ and $\beta_{\text {theo }}$ is observed whatever the value of $\varepsilon$, which demonstrates the validity of Eq. (11).

To conclude this study of the first regime, we point out that combining high-speed imaging and time-frequency analysis, we have demonstrated that Helmholtz's model applied to the bubble assumed as preserving its initial volume quantitatively describes the measured frequency chirp provided that the film opening dynamics is taken into account. As an illustration of the accuracy of this theoretical description of the bubble acoustic emission, we have superimposed on the spectrogram shown in Fig. $5(\mathrm{c})$ the prediction of $f_{H}$ based on Eqs. (11), (13), (8), which involves no free parameter.

As a final remark, we note that in the second regime (after $\tau_{b}$ ) $f_{0}$ actually still increases but much more slowly than in the first regime, as shown in Fig. 6 (a). Following Spiel [12], we attribute this slower chirp to the recession of the upper edge of the cavity, which is much slower (an order of magnitude less) than the film opening, as shown in Fig. 4 (b). 
Next, we test the quantitative validity of Eq. (11) by plotting the variation versus $R$ of $f_{0}$ measured at the end of the chirp (i.e. at $t=\tau_{b}$, see Fig. $6(\mathrm{a})$ ) versus $f_{H}\left(t_{b}\right)$ defined by Eq. (13) and computed using $\varepsilon=8 /(3 \pi)$ and $\varepsilon=16 /(3 \pi)$. Given the experimental scatter, an good agreement between $f_{0}\left(\tau_{b}\right)$ and $f_{H}\left(t_{b}\right)$ is observed. For $R<1 \mathrm{~mm}, \varepsilon=8 /(3 \pi)$ results in a quantitative agreement with the measurements. These results are consistent with the results of the work of Spiel [12] (see Fig. 7 in [12]). Interestingly, Eqs. (1), (13) constitute a closed-form, valid expression for the $f_{H^{-}} V$ relationship that can be applied to any liquid-gas system.

Finally, reminding that a lumped-element model of acoustic resonator is valid only for sub-wavelength resonators, i.e. for $R / \lambda \ll 1$, we aim to test the consistency of Helmholtz's model of open resonator applied to the collapsing bubble. The variation of $\lambda$ versus $R$ is plotted in the inset of Fig. 6 (d). We observe that $\lambda \gg R$ in the entire range of investigated values of $R$, which a posteriori justifies the use of Helmholtz's model.

\section{Quality factor}

The acoustic signal can also be characterised by its duration and its amplitude. Here we focus on the second regime of the acoustic emission and in particular on the characteristic timescale of decrease of the signal amplitude. Assuming a linear relaxation of the free pressure oscillations in the acoustic resonator, we expect the pressure signal to satisfy $p(t)=$ $p_{\max } \exp (-t / \tau) \cos \left(\omega_{0} t+\phi\right)$, where $\omega_{0}=2 \pi f_{0} . \tau$ can be experimentally evaluated as being equal to the laps $\delta t_{e}$ between the instant $t_{\max }$ at which $p$ reaches its maximum $p_{\max }$ and the instant at which its envelope reaches $p_{\max } / e$, see Fig. $5(\mathrm{~b})$. The quality factor $Q$ of the resonator can be deduced from $\tau$ through:

$$
Q=\frac{1}{2} \omega_{0} \tau
$$

The variation of $Q$ deduced from Eq. (14) versus $R$ is plotted in Fig. 7 (a). Interestingly, the upper boundary of the data is horizontal, corresponding to $Q \simeq 10$. According to [20], under the assumption of negligible thermo-viscous dissipation, the quality factor $Q$ of the resonator is predicted to satisfy:

$$
Q=2 \pi \sqrt{\frac{L^{\prime 3} V}{S^{3}}}
$$


Using the same assumptions as above for adapting Helmholtz's model to the opening bubble, $Q$ has the following expression:

$$
Q=2 \pi \sqrt{\varepsilon^{3} \frac{\sqrt{3}}{2 \pi} \frac{\ell_{c}^{3}}{R^{3}}}
$$

The smaller $Q$, the shorter the acoustic emission. Fig. 6b shows $\delta t$ is smaller when SDSsurfactant is used. To qualitatively explain this, we note that $Q$ increases with $\ell_{c}$ as $\ell_{c}^{3 / 2}$ (see Eq. (16)) and that $\ell_{c}$ increases with $\sigma$ as $\sigma^{1 / 2}$. Thus, the lower the surface tension, the smaller Q. Eq. 16 computed using $\varepsilon=8 /(3 \pi)$ and $\varepsilon=16 /(3 \pi)$ is plotted in Fig. 7 (a). We observe that the experimental data do not agree the theoretical prediction, particularly for small values of $R$, except as regards the order of magnitude. We believe that the thermo-viscous dissipation occurring in the vicinity of the neck may explain the observed discrepancy. A shown in the inset of Fig. 7 (a), the characteristic thermal length / thermal boundary layer thickness $\delta_{\mathrm{th}}=\sqrt{2 D_{\mathrm{th}} / \omega}\left(D_{\mathrm{th}}=2 \cdot 10^{-5} \mathrm{~m}^{2} \cdot \mathrm{s}^{-1}\right.$ is the thermal diffusivity of air $)$ is indeed close to $r_{c}$ in the experimentally investigated range of values of $R$, particularly for small values of $R$. The same considerations hold for the characteristic viscous length / viscous boundary layer thickness $\delta_{\mathrm{v}}=\sqrt{2 \nu / \omega}\left(\nu=1.5 \cdot 10^{-5} \mathrm{~m}^{2} \cdot \mathrm{s}^{-1}\right.$ is the kinematic viscosity in air at ambient temperature).
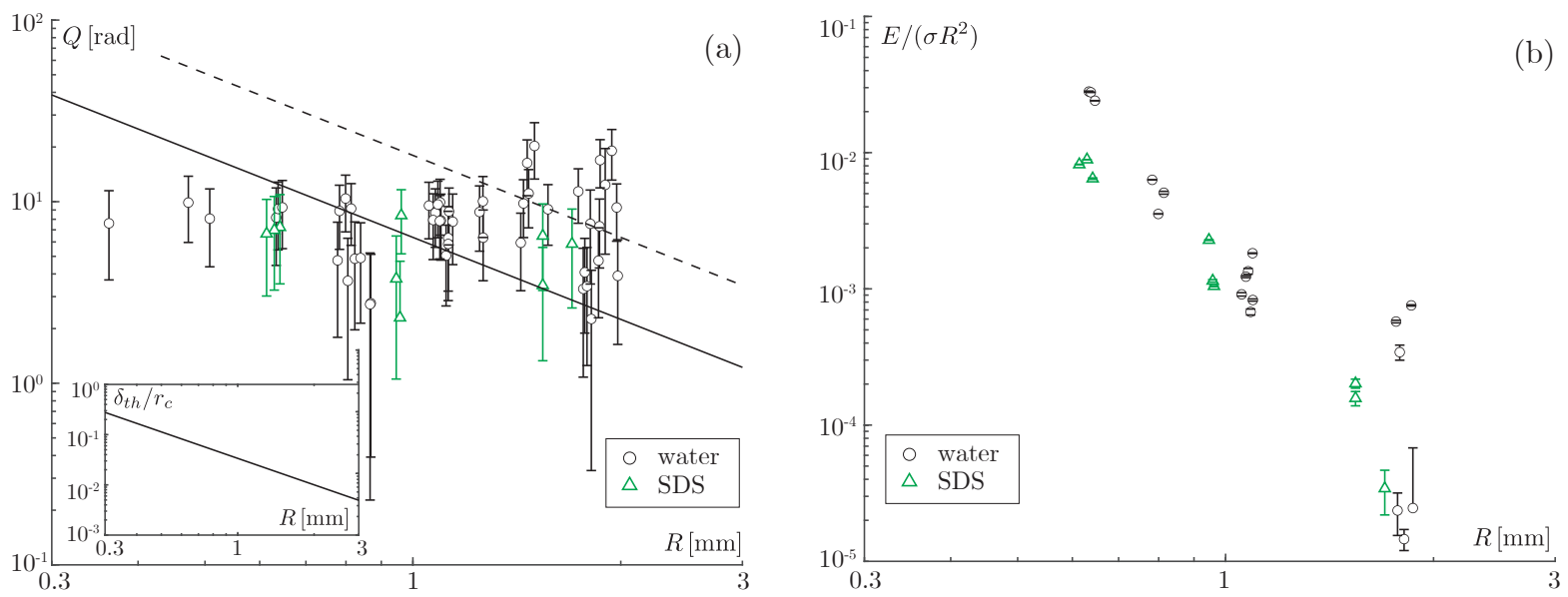

FIG. 7. (a) Measured quality factor $Q$ versus $R$. Solid line (respectively dashed line): theoretical prediction Eq. (16) computed using $\delta=8 /(3 \pi)$ (respectively $\delta=16 /(3 \pi)$. Inset: theoretical ratio of the characteristic thermal length to hole radius as function of bubble radius. (b) Dimensionless radiated acoustic energy $E /\left(\sigma R^{2}\right)$ versus $R$. 


\section{Radiated acoustic energy}

Since a collapsing bubble radiates sound that is characterised by a wavelength much larger than the bubble aperture, diffraction tends to scatter sound in all directions, and the microphone lies in the far-field of the bubble $\left(d \gg z\right.$, where $z \approx r_{c}^{2} / 2 \lambda$ is the Rayleigh distance). Thus, following the work of Divoux [21], we can assume an acoustic radiation with spherical symmetry and the total acoustic energy $E$ can be computed from the pressure signal $p(t)$ using :

$$
E=\frac{2 \pi d^{2}}{\rho_{g} c} \int_{0}^{\infty} p^{2}(t) d t
$$

Noting that the acoustic radiation originates from the sudden release of Laplace overpressure $\Delta P \simeq 2 \sigma / R$, we assume that $E$ is bounded by the energy stored in the floating bubble which scales as $\sigma R^{2}$. The variation of $E /\left(\sigma R^{2}\right)$ versus $R$ is plotted in Fig. 7 (b). We observe that(i) $E /\left(\sigma R^{2}\right)$ strongly decreases with $R$.

\section{CONCLUSION}

Let us summarise our results. Once a rising gas bubble has arrived at a free liquid surface, the liquid film separating the inner gas from the atmosphere drains and finally breaks when its thickness reaches a few micrometers, in absence or in presence of surfactants. The airborne acoustic emission associated with the release of the Laplace overpressure and the film opening start simultaneously. The frequency content of the resulting signal can be quantitatively explained using Helmholtz's model of an open resonator exhibiting a sequence of two regimes, (i) a first episode during which the liquid film opens and the resonator eigenfrequency drifts correspondingly (frequency chirp), (ii) a second regime characterised by a nearly constant oscillation eigenfrequency and a decreasing amplitude. At the end of the bubble acoustic emission, the liquid cavity has barely initiated its collapse. Interestingly, the acoustic emission of bubbles with radius smaller than $1.5 \mathrm{~mm}$ in water is audible in the first regime and is ultrasonic in the second regime.

Let us now come back to the champagne effervescence. Neglecting the first regime characterised by a frequency chirp, we can compute the central frequency of the acoustic emission of a collapsing champagne bubble with radius $R$ using Helmholtz's model of an open resonator Eq.(13) and the values of density and surface tension of champagne and of sound speed 
in $\mathrm{CO}_{2}$ given in Table I. From the histogram of the radii of floating champagne bubbles shown in Fig. 1 (b), we can compute the histogram of the corresponding acoustic resonant frequency, which is shown in Fig. 8. We note that this histogram quantitatively fits the acoustic peak-shaped spectrum of airborne acoustic emission of champagne except along its low-frequency tail. Moreover, both the average frequency of the histogram $\left\langle f_{H}\right\rangle=53 \mathrm{kHz}$ and the frequency of its maximum coincide with the spectrum average frequency. Since the the first regime of acoustic emission is characterised by frequencies smaller than those predicted using Eq. (13), we expect the low-frequency tail of the spectrum to correspond to the first regime that is precisely not modelled here. We stress that in the case of champagne most of the acoustic energy is in the ultrasonic regime.

We conclude that we have modelled the frequency content of the airborne acoustic emission of gas bubbles collapsing at a free liquid surface and in particular of champagne and sparkling liquids. When the film is completely retracted, we highlighted a closed-form expression of the resonance frequency at this time involving only one single geometric parameter, the gas volume. We believe that this quantitative description could be used to synthesise artificial acoustic signals of digital animation films as in the work of Langlois et al. [22].

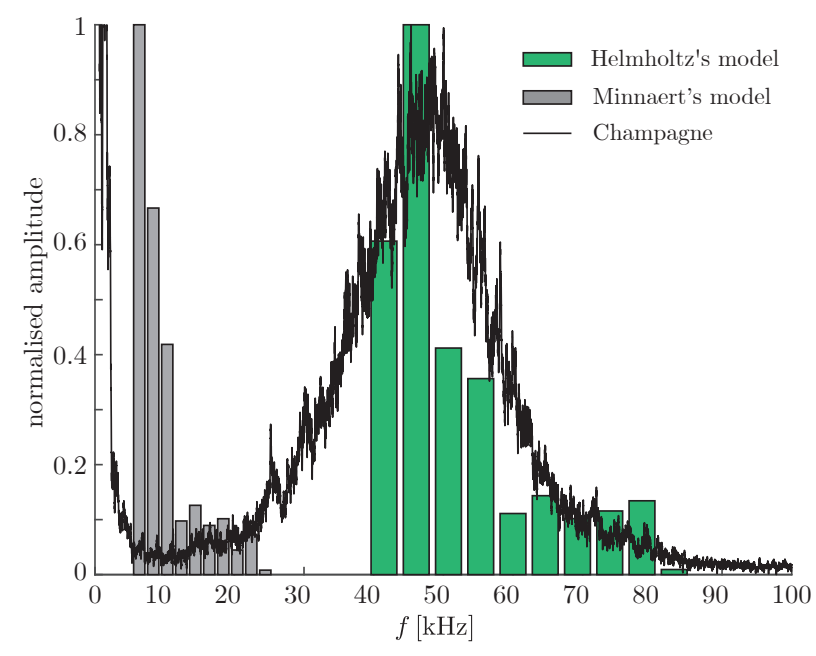

FIG. 8. Black solid curve: acoustic spectrum of the 1 s-duration airborne acoustic recording above a glass of champagne. Green bars: histogram of the resonant frequency of acoustic emission of the floating bubbles whose histogram of radii is shown in Fig. Fig. 1 (b) predicted using Helmholtz's model of open resonator Eq.(13). Grey bars: histogram of the resonant frequency of acoustic emission of the floating bubbles predicted using Minnaert's model of open resonator Eq. (18). 
According to Minnaert [23], subsurface gas bubbles undergoing volume oscillations are known to emit sound. The resonant frequency $f_{M}$ of their small amplitude, harmonic volume oscillations satisfies:

$$
f_{M}=\frac{1}{2 \pi R} \sqrt{\frac{3 \gamma P_{0}}{\rho_{\ell}}}
$$

where $\gamma$ is the gas adiabatic index $\left(\gamma=1.3\right.$ for $\left.\mathrm{CO}_{2}\right)$ and $P_{0}$ the atmospheric pressure. This equation is used to determine the radius distribution of submerged oscillating bubbles from the acoustic spectrum in studies of sparkling liquids [6], of the breaking of waves [24, 25], or of the sound emission of rain impact [26].

Actually, the process of airborne acoustic emission accompanying the bubble collapse markedly differs from Minnaert's model of acoustic emission of subsurface bubbles undergoing volume oscillations [23]. Consequently, $f_{M}$ markedly differs from $f_{H}$. To illustrate this, we have computed the histogram of the acoustic resonant frequency using Eq. (18) from the histogram of the radii of floating champagne bubbles shown in Fig. 1 (b). It is plotted in Fig. 8. We note that this histogram, whose average frequency is $\left\langle f_{M}\right\rangle=11 \mathrm{kHz}$, corresponds to a trough in the spectrum of airborne acoustic emission of champagne. We conclude that this mechanism of sound radiation does not contribute to the airborne acoustic emission of champagne.

More generally, this work is a new step in understanding the acoustic signature of violent hydrodynamic events, which adds to previous studies on volcano eruptions [21, 27, 28], impacts on liquid surfaces by droplets [26] or solid spheres [29], breaking waves [24, 25] or bursting soap bubbles [30]. We believe that the analysis of the acoustic signature of such fast and violent hydrodynamic events should provide information, complementary to highspeed imaging, on the main flow features, on the forces at play and on the relying physical processes. 


\section{ACKNOWLEDGEMENT}

We thank Caroline Derec, Valentin Leroy and Adrien Bussonnière for fruitful discussions.

[1] G. Liger-Belair, The physics and chemistry behind the bubbling properties of Champagne and sparkling wines: A state-of-the-art review, J. Agri. Food Chem. 53, 8 (2005)

[2] G. Liger-Belair et al.,Unraveling different chemical fingerprints between a champagne wine and its aerosols, Proc. Natl Acad. Sci. USA 106, 39 (2009)

[3] G. Liger-Belair and T. Séon and A. Antkowiak, Collection of collapsing bubble driven phenomena found in champagne glasses, Bubble Science, Engineering and Technology 4, 1 (2012)

[4] E. Ghabache, G. Liger-Belair, A. Antkowiak and T. Séon, Evaporation of droplets in a Champagne wine aerosol, Scientific Reports 6, 25148 (2016)

[5] G. Liger-Belair et al., Kinetics of Gas Discharging in a Glass of Champagne: The Role of Nucleation Sites, Langmuir 18, 4 (2002)

[6] K. Spratt, K. Lee and P. Wilson, Champagne acoustics, Physics Today 71, 8 (2008).

[7] R. Span and W. Wagner, A new equation of state for carbon dioxide covering the fluid region from the triple-point temperature to $1100 \mathrm{~K}$ at pressures up to $800 \mathrm{MPa}$, J. Phys. Chem. ref. Data 25, 1509 (1996)

[8] L. Duchemin and S. Popinet and C. Josserand and S. Zaleski, Jet formation in bubbles bursting at a free surface, Phys. Fluids 14, 9 (2002)

[9] E. Ghabache, A. Antkowiak, C. Josserand and T. Séon, On the physics of fizziness: How bubble bursting controls droplets ejection, Phys. Fluids 26, 12 (2014)

[10] L. Deike et al., Dynamics of jets produced by bursting bubbles, Physical Review Fluids 3, 013603 (2018)

[11] A. Gañán-Calvo, Revision of Bubble Bursting: Universal Scaling Laws of Top Jet Drop Size and Speed, Phys. Rev. Lett. 119, 204502 (2017)

[12] D. Spiel, Acoustical Measurements of Air Bubbles Bursting at a Water Surface: Bursting Bubbles as Helmholtz Resonators, J. Geophys. Res. 97, C7 (1992)

[13] G. Deane, Determining the bubble cap film thickness of bursting bubbles from their acoustic emissions, JASA 133, 2 (2013) 
[14] Y. Toba, Drop Production by Bursting of Air Bubbles on the Sea Surface (II) Theoretical Study on the Shape of Floating Bubbles, Journal of the Oceanographical Society of Japan 15, $3(1959)$

[15] H. Lhuissier and E. Villermaux, Bursting bubble aerosols, JFM 696, 5-44 (2012)

[16] L. Champougny, M. Roché, W. Drenckhan and E. Rio, Life and death of not so "bare" bubbles, Soft matter 12, $24(2016)$

[17] G. Taylor, The dynamics of thin sheets of fluid. III. Disintegration of fluid sheets, Proc. R. Soc. London A 253, 1274 (1959)

[18] F. Culick, Comments on a ruptured soap film, J. Appl. Phys. 31, 6 (1960)

[19] W. McEntee and K. Mysels, Bursting of soap films. I. An experimental study, J. Phys. Chem. 73, 9 (1969)

[20] L. Kinsler, A. Frey, A. Coppens and J. Sanders, Fundamentals of acoustics (Wiley), ed. 4 (2000)

[21] T. Divoux, V. Vidal, F. Melo and J.-C. Géminard, Acoustic emission associated with the bursting of a gas bubble at the free surface of a non-Newtonian fluid, Phys. Rev. E 77, 056310 (2008)

[22] TR. Langlois, C. Zheng and DL. James, Toward animating water with complex acoustic bubbles, ACM Trans. Graphics (TOG), 35, 95 (2016)

[23] M. Minnaert, On musical air-bubbles and the sounds of running water, The London, Edinburgh, and Dublin Philosophical Magazine and Journal of Science 16, 104 (1933)

[24] K. Bolin and M. Åbom, Air-borne sound generated by sea waves, JASA 127, 5 (2010)

[25] G. Deane, M. Stokes, Scale dependence of bubble creation mechanisms in breaking waves, Nature 418, 6900 (2002)

[26] A. Prosperetti and H. Oguz, The Impact of Drops on Liquid Surfaces and the Underwater Noise of Rain, Annual Review of Fluid Mechanics 25, 1 (1993)

[27] S. Vergniolle and M. Boichu and J. Caplan-Auerbach, Acoustic measurements of the 1999 basaltic eruption of Shishaldin volcano, Alaska: 1. Origin of Strombolian activity, Volcanol. Geotherm. Res. 137, 1-3 (2004)

[28] V. Vidal et al., Dynamics of soap bubble bursting and its implications to volcano acoustics, Geophysical Research Letters 37 , 7 (2010) 
[29] C. Duez, C. Ybert, C. Clanet and L. Bocquet, Making a splash with water repellency, Nature physics 3, 3 (2007)

[30] A.Bussonnière et al., Acoustic Sensing of Forces Driving Fast Capillary Flows, Phys. Rev. Lett., 124, $084502(2020)$ 